\title{
Otimização de Carteiras baseada em Modelos de Correlações Condicionais
}

\section{Portfolio Optimization based on Conditional Correlation Models}

André Alves Portela Santos*

\begin{abstract}
Resumo: Modelos multivariados de volatilidade baseados em correlações condicionais têm recebido grande atenção em função da sua capacidade de modelar a dinâmica presente nas matrizes de covariâncias de retornos de ativos financeiros. Neste artigo, analisa-se o desempenho dessa classe de modelos quando aplicados ao problema de seleção de otimização de carteiras de ações. Para isso, implementam-se quatro diferentes especificações parcimoniosas de modelos de correlações condicionais da família Garch. Utilizando-se dados dos mercados acionários brasileiro e norteamericano, obtêm-se carteiras de mínima variância para cada um dos mercados. Os resultados indicam que as carteiras de variância mínima obtidas com modelos de correlações condicionais obtiveram resultados superiores em termos de menor risco, maior retorno ajustado ao risco, maior retorno bruto acumulado e maior excesso de retorno acumulado quando comparados aos principais índices de referência de cada um dos países.
\end{abstract}

Palavras-chave: Índice de Sharpe. Otimização de carteiras. Volatilidade.

Abstract: Multivariate volatility models based on conditional correlations have been receiving increased attention due to the possibility of modeling the dynamics in the covariance matrices of financial asset returns. In this paper, the performance of this class of models is analyzed when applied to the problem of optimal portfolio selection of a portfolio of stocks. For that purpose, it is implemented four alternative parsimonious specifications for the class of conditional correlation Garch models. Using data from the Brazilian and North-American stock markets, it is obtained optimal minimum variance portfolios for each of the markets. The results indicate that the minimum variance portfolios obtained with conditional correlation models achieved superior results in terms of lower risk, higher risk-adjusted returns, higher cumulative gross returns and higher cumulative excess returns when compared to the main stock indices of each market.

Keywords: Sharpe index. Portfolio optimization. Volatility.

JEL Classification: G11; G14; G17.

Doutor em Finanças Quantitativas pela Universidad Carlos III de Madrid. Professor do Departamento de Economia da Universidade Federal de Santa Catarina (UFSC). E-mail: andre. portela@ufsc.br 


\section{1 lntrodução}

Nas últimas décadas tem havido um notável crescimento no uso da modelagem financeira e de ferramentas de otimização para o gerenciamento de carteiras de ativos financeiros. Boa parte dessas ferramentas está construída sob o paradigma da análise média variância introduzida por Markowitz (1952), a qual transformou o processo de alocação de ativos em um problema de otimização com base no trade-off fundamental entre retorno esperado e risco. Dessa forma, o investidor deve escolher a carteira com a menor variância entre um infinito número de carteiras que proporcionem um determinado retorno, ou, de forma equivalente, para um determinado nível de aversão ao risco, escolher a carteira que maximize o retorno esperado.

Conforme observam Caldeira, Moura e Santos (2013), a implementação da otimização de carteiras com base no critério média variância esbarra na dificuldade de se obter estimações acuradas dos retornos esperados dos ativos e da matriz de covariâncias desses retornos. Via de regra, essas estimativas estão sujeitas a erros de estimação, o que prejudica o desempenho da carteira otimizada com base nesses estimadores. Além disso, a estimação de retornos esperados está sujeita a um maior erro de estimação em comparação à estimação de covariâncias, e o erro de estimação nos retornos esperados tem um impacto negativo maior no desempenho da carteira quando comparado ao impacto do erro de estimação nas covariâncias. Em vista disso, a pesquisa acadêmica recente tem focado em carteiras de mínima variância, as quais dependem somente da estimação de covariâncias e estão sujeitas a uma quantidade menor de erro de estimação em comparação às carteiras de média variância (JAGANNATHAN; MA, 2003). Nesse caso, o investidor importa-se apenas com a minimização do risco da carteira, sendo necessária a estimação de uma matriz de variâncias e covariâncias entre os retornos dos ativos. De fato, uma extensa lista de referências tem apontado que carteiras de mínima variância possuem um desempenho fora da amostra melhor que quaisquer carteiras de média variância, mesmo quando o critério de desempenho considera tanto o retorno da carteira quanto também seu risco. ${ }^{1}$

Vale observar que estudos anteriores avaliaram o desempenho de carteiras otimizadas utilizando dados do mercado acionário brasileiro. O trabalho de Tomé et al. (2011), por exemplo, propõe a construção de índices de variância mínima para o Brasil. Os autores concluem que o índice construído com base em carteiras de variância mínima irrestritas não consegue um desempenho superior ao índice de referência. Entretanto, os resultados das carteiras otimizadas melhoram à medida que uma restrição de alocação máxima em cada ativo é colocada. Santos e

1 A esse respeito ver, por exemplo, Ledoit e Wolf (2004), Engle, Shephard e Sheppard (2008), Santos e Moura (2014), Santos e Tessari (2012) e Caldeira, Moura e Santos (2013). 
Tessari (2012) analisam o desempenho fora da amostra de carteiras de variância mínima construídas para o mercado acionário brasileiro e concluem que essa política de investimento proporcionam resultados estatisticamente significativos em termos de menor volatilidade e desempenho ajustado ao risco superior. Rubemsam e Beltrame (2013) demonstram que carteiras de variância mínima superam vários benchmarks em termos de retornos e volatilidade. Por fim, Caldeira et al. (2014) demonstram que a política de investimentos baseada em carteiras de variância mínima também pode ser aplicada para a construção de portfólios ótimos compostos por fundos de investimentos multimercado. Os portfólios de fundos resultantes superam benchmarks de mercado tanto nas áreas de renda fixa quanto de renda variável.

A obtenção das carteiras de variância mínima esbarra ainda no problema da dimensionalidade, uma vez que é frequente a necessidade de se estimar matrizes de covariâncias para um grande número de ativos. Conforme observam Fleming, Kirby e Ostdiek (2001), essas matrizes eram inicialmente especificadas segundo a matriz de covariâncias incondicional (ou amostral). Entretanto, os autores mostram que modelos para matrizes de covariâncias condicionais têm significante valor econômico quando comparados à otimização de carteiras com base na matriz de covariâncias incondicional, em função de sua maior capacidade de capturar a dinâmica no segundo momento condicional dos retornos de ativos financeiros. A maioria das tentativas iniciais de se construir modelos para as covariâncias condicionais, como, por exemplo, o modelo Vech de Bollerslev, Engle e Wooldridge (1988), e o modelo Bekk, de Engle e Kroner (1995), entre outros, sofre da chamada maldição da dimensionalidade. Nessas especificações, o número de parâmetros cresce rapidamente à medida que a dimensão do problema aumenta, criando dificuldades no processo de estimação e introduzindo erro de estimação nas matrizes de covariâncias estimadas. Nesse contexto, os modelos de correlações condicionais introduzidos por Bollerslev (1990) emergem como alternativas mais parcimoniosas e que requerem uma quantidade menor de parâmetros a serem estimados. ${ }^{2}$ A principal atratividade dos modelos de correlações condicionais é a possibilidade de estimar separadamente variâncias univariadas e a matriz de correlações. Essa decomposição da matriz de covariâncias em variâncias e correlações simplifica a estimação e torna o modelo mais flexível e mais atrativo em relação às especificações Garch multivariadas mencionadas anteriormente, especialmente no que se refere à possibilidade de sua utilização em problemas que envolvem um grande número de séries temporais. Além disso, a evidência internacional sugere que modelos de correlações condicionais possuem êxito quando aplicados ao problema de otimização de carteiras. Engle e Colacito (2006), por exemplo, demonstram que o modelo de correlações condicionais dinâmicas e assimétricas supera um amplo

$2 \quad$ Ver Silvennoinen e Terasvirta (2009). 
conjunto de benchmarks quando aplicado ao problema de obtenção de carteiras otimizadas para o mercado norte-americano.

O objetivo deste artigo é implementar e avaliar o desempenho de modelos Garch multivariados da classe de correlações condicionais para a obtenção de carteiras de variância mínima envolvendo um grande número de ativos. Para isso, são implementados o modelo de correlações condicionais constantes (CCC-GAR$\mathrm{CH}$ ), de Bollerslev (1990), o modelo de correlações condicionais dinâmicas (DCC-GARCH), de Engle (2002), o modelo de equicorrelações condicionais dinâmicas (DECO-GARCH), de Engle e Kelly (2012) e o modelo correlações condicionais assimétricas (ASYDCC-GARCH), de Cappiello, Engle e Sheppard (2006). Como forma de avaliar a robustez dos resultados, são utilizados dois conjuntos de dados distintos. O primeiro conjunto de dados contém as 45 ações de maior liquidez negociadas no mercado acionário brasileiro, enquanto o segundo é composto por 45 ativos de maior liquidez e constituintes do índice SEP100 do mercado acionário norte-americano. A avaliação do desempenho das carteiras de mínima variância é feita com base nas medidas de turnover, índice de Sharpe, média do excesso de retorno, desvio padrão dos retornos, retorno bruto acumulado, excesso de retorno acumulado e custo de transação breakeven (HAN, 2006). O desempenho comparativo das carteiras otimizadas é feito em relação aos índices de ações Ibovespa e SEP100 dos mercados brasileiro e norte-americano, respectivamente. Além disso, é utilizada a taxa CDI como proxy da taxa livre de risco para o mercado brasileiro e, para o mercado norte-americano, o retorno dos títulos da dívida dos Estados Unidos (T-Bill) com vencimento de um mês. Os resultados encontrados no artigo depõem favoravelmente ao uso dos modelos multivariados de volatilidade. Foi verificado que o risco das carteiras de variância mínima obtida com os modelos multivariados de volatilidade foi substancialmente e estatisticamente inferior ao obtido pelos índices de referência de ambos os mercados. Além disso, o retorno ajustado ao risco das carteiras de mínima variância obtida com algumas das especificações adotadas no artigo foi estatisticamente superior àquela obtida pelo índice de mercado usado como referência.

É importante ressaltar que este artigo traz extensões importantes em relação à literatura de otimização de carteiras para o mercado brasileiro. Primeiro, considera um conjunto diferente de especificações para a matriz de covariâncias condicional em comparação àquelas adotadas nos trabalhos de Tomé et al. (2011), Santos e Tessari (2012), Rubesam e Beltrame (2013) e Caldeira et al. (2014). Mais especificamente, Rubesam e Beltrame (2013) consideram a especificação DCC-GARCH para a obtenção da matriz de covariâncias condicional. Neste trabalho, entretanto, considera-se um conjunto mais amplo de modelos de correlações condicionais e demonstra-se que algumas dessas especificações superam os resultados obtidos pelo modelo DCC-GARCH adotado em Rubesam e Beltrame (2013). Se- 
gundo, o artigo faz uma análise mais ampla do desempenho da política de investimento baseada em carteiras de variância mínima ao considerar dados dos mercados acionários brasileiros e norte-americano, o que aumenta consideravelmente a robustez dos resultados.

Este artigo está organizado da seguinte forma: a seção 2 traz uma revisão das principais especificações Garch multivariadas encontradas na literatura, além das especificações adotadas no artigo; a seção 3 descreve a metodologia de obtenção e avaliação das carteiras de variância mínima; a seção 4 descreve os resultados encontrados; e, para finalizar, a seção 5 traz uma síntese conclusiva.

\section{Modelos Multivariados da Familia Garch}

Esta seção dedica-se a rever alguns dos modelos multivariados de volatilidade da classe Garch mais relevantes. O desafio que enfrenta a literatura dos modelos da família Garch multivariados consiste no número de parâmetros a serem estimados. Modelos bem simples em termos de generalidade podem rapidamente se mostrar intratáveis quando alguns ativos são adicionados ou uma estrutura temporal um pouco mais sofisticada é proposta. A especificação do modelo Garch multivariado é dada por:

$$
y_{t}=\varepsilon_{t} H^{\frac{1}{2}}
$$

na qual $\varepsilon_{\mathrm{t}}$ representa um processo de dimensão 1 x $N$ com média zero e matriz de covariâncias dada por uma matriz identidade de ordem $N x N$, em que $N$ é o número de ativos envolvidos. A distribuição de $y_{t}$ condicionada à informação passada, denotada por $I_{t-1}$, satisfaz as seguintes propriedades: $E\left[y_{t} \mid I_{t-1}\right]=0$ e $E\left[y_{t} y_{t}^{\prime} \mid I_{t-1}\right]=H_{t^{\prime}}$ em que $H_{t}$ é uma matriz de covariâncias positiva definida.

O modelo Vech, proposto por Bollerslev, Engle e Wooldridge (1988), é uma generalização do modelo de Garch univariado no qual a matriz de covariância condicional $H_{t}$ depende de suas próprias defasagens e também das defasagens do produto cruzado dos retornos. Dessa forma, o modelo pode ser escrito da seguinte maneira:

$$
\operatorname{vech}\left(H_{T}\right)=C C^{\prime}+\sum_{j=1}^{q} A_{i} \operatorname{vech}\left(y_{t-j} y_{t-j}^{\prime}\right)+\sum_{j=1}^{q} B_{j} \operatorname{vech}\left(H_{t-j}\right),
$$

em que vech é o operador que transforma a parte triangular inferior da matriz $H_{t}$ em um vetor coluna; $A$ e $B$ são matrizes de parâmetros; e $C$ é uma matriz de parâmetros triangular inferior. Esse modelo é extremamente genérico e flexível. Entretanto, o número de parâmetros que devem ser estimados cresce exponencialmente à medida que cresce o número de ativos envolvidos no problema, o 
que inviabiliza sua aplicação em problemas de grande dimensão. Bollerslev, Engle e Wooldridge (1988) propõem uma versão mais parcimoniosa do modelo Vech, no qual $A_{i}$ e $B_{j}$ são matrizes diagonais. Dessa forma, o número de parâmetros que devem ser estimados reduz-se significativamente.

O modelo Bekk, proposto por Engle e Kroner (1995), é uma variação do modelo Vech e pode ser escrito como:

$$
H_{t}=C C^{\prime}+\sum_{j=1}^{q} A_{j} r_{t-j} r_{t-j}^{\prime} A_{j}+\sum_{j=1}^{p} B_{j} H_{t-1} B_{j},
$$

em que as matrizes de parâmetros $A$ e $B$ podem ser completas, diagonais ou escalares. A atratividade desse modelo reside no fato de que a positividade da matriz $H_{t}$ é imediatamente garantida em função da especificação sob forma quadrática dos termos $\sum_{j=1}^{q} A_{j} r_{t-j} r_{t . j}^{\prime} A_{j}$ e $\sum_{j=1}^{p} B_{j} H_{t-1} B_{j}$. Entretanto, o modelo na sua forma completa ou diagonal possui uma grande quantidade de parâmetros estimados, o que reduz a sua atratividade para problemas de grande dimensão.

Uma série de especificações alternativas aos modelos Vech e Bekk tem sido propostas com o objetivo de se alcançar versões mais parcimoniosas que requeiram um número reduzido de parâmetros e que possibilitem seu uso em problemas envolvendo um grande número de séries temporais. Um dessas abordagens é o modelo de fatores proposto por Engle, Ng e Rothschild (1990). Essa classe de modelos é construída a partir da observação empírica de que alguns fatores latentes comuns possuem poder explicativo sobre o comportamento dessas variáveis. Nesse modelo, assume-se que as observações são geradas por fatores condicionalmente heterocedásticos que possuem uma estrutura do tipo Garch. O modelo pode ser escrito como:

$$
H_{t}=\Omega+\sum_{k=1}^{k} w_{k} w_{k}^{\prime} f_{k, t}
$$

em que $\Omega$ é uma matriz positiva semidefinida; $\mathrm{w}_{\mathrm{k}}, \mathrm{k}=1, \ldots, \mathrm{K}$ é um vetor dos pesos linearmente independentes; e $f_{k, t}$ são os fatores comuns. Supõe-se que esses fatores seguem uma dinâmica Garch do tipo:

$$
f_{k, t}=\omega_{k}+\alpha_{k}\left(\gamma_{k}^{\prime} y_{t-1}\right)^{2}+\beta_{k} f_{k, t-1}
$$

em que $\omega_{k}, \alpha_{k}$ e $\beta_{k}$ são escalares e $\gamma_{k}$ é o vetor dos pesos. Um dos problemas com esses modelos é que geralmente os fatores que afetam as variáveis são correlacionados, podendo gerar uma indesejável característica que seria os fatores capturarem características semelhantes. Assim, são propostas várias especificações alternativas que gerem fatores não correlacionados, como, por exemplo, o modelo Garch ortogonal generalizado de Van der Weide (2002), entre outros. 
Neste artigo, adota-se outra classe de modelos da família Garch com uma especificação mais parcimoniosa em relação aos modelos Vech e Bekk: os modelos de correlações condicionais. Essa classe de modelos tem ganhado notoriedade na literatura e é baseada na decomposição da matriz de covariâncias condicionais em uma matriz diagonal de variâncias condicionais e uma matriz de correlação, as quais podem ser obtidas separadamente. $\mathrm{O}$ modelo de correlações condicionais pode ser escrito como:

$$
H_{t}=D_{t} P D_{t^{\prime}}
$$

em que $\mathrm{D}_{\mathrm{t}}=\operatorname{diag}\left(h_{1 t}{ }^{1 / 2}, \ldots, h_{\mathrm{Nt}}{ }^{1 / 2}\right)$ é uma matriz diagonal na qual os elementos da diagonal principal são as volatilidades condicionais de cada um dos ativos especificados através de modelos Garch univariados; e $P$ é uma matriz de correlações positiva definida.

A matriz de covariâncias especificada na equação 6 pode ser especificada de forma hierárquica. Primeiro, é escolhido um modelo para modelar a variância de cada ativo, de modo a preencher os elementos da diagonal principal da matriz de $D_{t}$. O segundo passo é estabelecer uma especificação para a matriz de correlações P. Bollerslev (1990) propõe uma especificação na qual as correlações condicionais são constantes e, assim, as covariâncias condicionais são proporcionais ao produto das volatilidades condicionais correspondentes. Essa restrição reduz significativamente o número de parâmetros desconhecidos e, assim, simplifica a estimação. Esse modelo foi batizado de modelo de correlações condicionais constantes ou CCC-GARCH (constant conditional correlation Garch).

A suposição de que as correlações condicionais são constantes pode ser relaxada. Engle (2002) e Tse e Tsui (2002) propõem uma generalização do modelo CCC-GARCH, tornando a matriz de correlação condicional dependente do tempo. O modelo é então chamado de correlação condicional dinâmica, ou modelo DCC-GARCH (dynamic conditional correlation Garch). Neste artigo, adotou-se o modelo DCC-GARCH proposto por Engle (2002), o qual é especificado como:

$$
H_{t}=D_{t} P_{t} D_{t}
$$

em que $P_{t}$ é uma matriz de correlações condicionais simétrica positiva definida com elementos $\rho_{\mathrm{i}, \mathrm{t}}$ em que $\rho_{\mathrm{i}, t}=1, i, j=1, \ldots, N$ e $\rho_{\mathrm{i}, \mathrm{t}}$ é dado por

$$
\rho_{i j, t}=\frac{q_{i i, t}}{\sqrt{q_{i i, t} q_{j j, t}}},
$$

em que $q_{i j, t}$ são agrupados na matriz $Q_{t}$ de dimensão $N \times N$, a qual segue um processo Garch: 


$$
Q_{t}=(\bar{P}-a \bar{P}-b \bar{P})+a \varepsilon_{t-1} \varepsilon_{t-1}^{\prime}+b Q_{t-1},
$$

no qual $\varepsilon_{t}=y_{t} / \sqrt{ } h_{t}$ e $a$ e $b$ são parâmetros escalares que satisfazem $a+b<1$, e $\bar{P}$ é a matriz de correlações incondicional.

O modelo DCC-GARCH de Engle (2002) foi estendido por Cappiello, Engle e Sheppard (2006) de modo a permitir a inclusão de efeitos assimétricos advindos de notícias positivas e negativas sobre as correlações condicionais. O modelo é especificado como:

$$
Q_{t}=(\bar{P}-a \bar{P}-b \bar{P}-g \bar{N})+a \varepsilon_{t-1} \varepsilon_{t-1}^{\prime}+b n_{t-1} n_{t-1}^{\prime}+g Q_{t-1},
$$

no qual $a, b$ e g são parâmetros escalares e $\mathrm{n}_{\mathrm{t}}=\mathrm{I}\left[\varepsilon_{\mathrm{t}}<0\right] \odot \varepsilon_{\mathrm{t}}$, em que I é uma função indicadora que identifica retornos negativos e $\odot$ é o produto Hadamard (elemento por elemento). A condição para a ocorrência da positividade definida é a de que $\mathrm{a}^{2}+\mathrm{b}^{2}+\delta \mathrm{g}^{2}<1$, na qual $\delta$ é o máximo autovalor de $\left[\bar{P}^{-12} \bar{N} \bar{P}^{-12}\right]$, em que $\bar{N}=\mathrm{E}\left[\mathrm{n}_{\mathrm{t}} \mathrm{n}_{\mathrm{t}}^{\prime}\right]$. Esse modelo foi batizado de modelo de correlações condicionais dinâmicas assimétricas, ou modelo ASYDCC-GARCH (asymmetric dynamic conditional correlation Garch).

Alternativamente, a matriz de correlação condicional $P_{t}$ na equação 7 pode ser modelada com mais parcimônia, utilizando uma especificação dynamic equicorrelation (DECO-GARCH) proposta por Engle e Kelly (2012), a qual assume que todas as correlações condicionais dinâmicas são iguais. Nesse caso, tem-se que

$$
P_{t}=P_{t}^{D E C O},
$$

em que $P_{t}^{D E C O}$ é a matriz de equicorrelação condicional definida como

$$
P_{t}^{D E C O}=\left(1-P_{t}^{e q u i}\right) I_{n}+P_{t}^{e q u i} J_{n},
$$

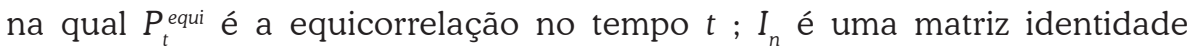
$N$-dimensional; e $J_{n}$ é uma matriz $N \times N$ de 1 .

\section{Seleção e Otimização de Carteiras de lnvestimento}

A otimização com base na relação média variância proposta inicialmente por Markowitz (1952) é a abordagem padrão para a construção de carteiras ótimas. A suposição básica por trás desse modelo é a de que as preferências de um investidor podem ser representadas por uma função (função de utilidade) dos retornos esperados e da variância da carteira. Santos (2010) ressalta que a escolha entre o prêmio de risco desejado (retorno esperado acima da taxa livre de risco) depende da tolerância do investidor ao nível de risco. Indivíduos menos avessos ao risco 
podem estar dispostos a aceitar uma maior volatilidade em suas carteiras a fim de alcançar um maior prêmio de risco, enquanto investidores avessos ao risco preferirão carteiras menos voláteis, penalizando, portanto, o retorno esperado. Para incorporar o trade-off ótimo entre retorno esperado e risco, considera-se o problema enfrentado por um investidor que deseja alocar sua riqueza entre $N$ ativos de risco, procurando saber que peso $\mathrm{w}_{\mathrm{i}}$ deve dar a cada ativo de maneira a atingir $\mathrm{o}$ menor nível de risco para um dado nível de retorno esperado, conforme desenvolvido por Brandt (2010). A escolha do investidor está representada em um vetor $N \times 1$ de pesos, $\mathrm{w}=\left(\mathrm{w}_{1}, \ldots, \mathrm{w}_{N}\right)^{\prime}$, no qual cada peso $\mathrm{w}_{i}$ representa o percentual do i-ésimo ativo mantido na carteira. Supõe-se que a carteira é totalmente investida, isto é, $\sum_{i=1}^{N} \mathrm{~W}_{i}=1, e \mathrm{w}_{\mathrm{i}} \geq 0$, o que significa que não é permitido venda a descoberto. ${ }^{3}$

Considerando os $N$ ativos de risco com vetor de retorno aleatório $R_{t+1}$, o retorno da carteira de $t$ a $t+1$ é dado por $R_{p, t+1}=\sum_{i=1}^{N} \mathrm{~W}_{\mathrm{i}, t} R_{\mathrm{i}, t+1}=\mathrm{w}^{\prime} R$, em que $R_{p, t+1}$ está condicionado aos pesos conhecidos em t. Supõe-se $R_{t} \sim N\left(\mu_{t}, H_{p}\right)$, com $\mu_{\mathrm{t}}=\left\{\mu_{1, \mathrm{t}}, \ldots, \mu_{\mathrm{N}, \mathrm{t}}\right\}$ e $H_{\mathrm{t}}=\left\{h_{\mathrm{i}, \mathrm{t}}\right\}$ respectivamente, o vetor de médias e a matriz de covariâncias. O retorno da carteira $R_{p, t}=\mathrm{w}_{\mathrm{t}} R_{\mathrm{t}}$ é normal com média $\mu_{\mathrm{t}}=\mathrm{w}_{\mathrm{t}}{ }_{\mathrm{t}} \mu_{\mathrm{t}} \mathrm{e}$ variância $h_{p, t}^{2}=\mathrm{w}_{t}^{\prime} H_{t} \mathrm{w}_{t}$. Dessa forma, o problema de alocação ótima de portfólio do investidor consiste em um problema de minimização restrita dado por

$$
\min _{w_{t}} w_{t}^{\prime} H_{t} w_{t}-\frac{1}{\gamma} E\left[R_{p, t+1}\right]
$$

sujeito a

$$
\begin{gathered}
I^{\prime} \mathrm{w}_{t}=1 \\
\mathrm{w}_{\mathrm{i}} \geq 0, \forall i=1, \ldots, N,
\end{gathered}
$$

em que w $\in \mathbb{R}^{N}$ é o vetor de pesos da carteira; $E\left[R_{p, t+1}\right]$ é a estimativa do retorno esperado da carteira; $\gamma$ é o parâmetro que mede o nível relativo de aversão ao risco; e $w_{i} \geq 0$ representa a restrição de venda a descoberto. A restrição 1 ' $w_{t}$, na qual $1 \in \mathbb{R}^{N}$ é um vetor cujos elementos são iguais a 1 e que garante que a soma dos pesos da carteira é um. Para diferentes valores do parâmetro de aversão ao risco, obtêm-se diferentes carteiras na fronteira eficiente.

A carteira de mínima variância corresponde a um caso especial da carteira de média variância com parâmetro de aversão ao risco infinito $(\gamma=\infty)$. Nesse caso, o investidor preocupa-se somente com o risco da carteira, e não com seu retorno esperado. Mais especificamente, a carteira de variância mínima pode ser obtida através da solução do problema:

3 O termo "venda a descoberto" refere-se à operação de venda de um ativo que não consta na carteira do investidor. 


$$
\min _{w t} w_{t} H_{t} w_{t}
$$

sujeito a

$$
I^{\prime} w_{t}=1
$$

A solução para o problema é dada por

$$
w_{t}=\frac{H_{t}^{-1}}{\iota^{\prime} H_{t}^{-1} \iota}
$$

Para o caso restrito a vendas a descoberto, adiciona-se ao problema de otimização definido nas equações 15 e 16 a restrição $w_{i} \geq 0, \forall i=1, \ldots, N$, ou seja, o peso de cada ativo na carteira não pode ser negativo. Essa imposição gera resultados melhores, principalmente em relação à redução nos custos de rebalanceamento das carteiras otimizadas. ${ }^{4}$ Nesse caso, o vetor de pesos ótimo é obtido através de métodos numéricos. ${ }^{5}$

Vale observar ainda que a literatura aponta que é mais difícil estimar médias (ou seja, retornos esperados) do que covariâncias dos retornos de ativos, e também que os erros das estimativas das médias têm um impacto maior sobre o peso do portfólio do que os erros nas estimativas de covariâncias. Por esse motivo, Jagannathan e Ma (2003) apontam que a pesquisa acadêmica recente tem se concentrado em portfólios de mínima variância, que dependem exclusivamente das estimativas de covariâncias e, portanto, são menos vulneráveis ao erro de estimação de média variância. Além disso, evidências empíricas mostram que a carteira de mínima variância tem melhor desempenho fora da amostra do que qualquer outra carteira de média variância, mesmo quando se utilizam medidas de desempenho que dependem tanto da média quanto da variância.

A avaliação do desempenho das carteiras de variância mínima é feira com base nos seguintes indicadores: média do retorno, desvio padrão, índice de Sharpe e turnover. Podemos escrever tais medidas como as seguintes equações:

$$
\begin{gathered}
\text { Retorno Médio }=\hat{\mu}=\frac{1}{L-T} \sum_{t=T}^{L-1} w_{t}^{\prime} R_{t+1} \\
\text { Desvio padrão }=\hat{\sigma}=\sqrt{\frac{1}{L-T-1} \sum_{t=T}^{L-1}\left(w_{t}^{\prime} R_{t+1}-\hat{\mu}\right)^{2}} \\
\text { Índice de Sharpe }=\frac{\widehat{\mu}}{\hat{\sigma}}
\end{gathered}
$$

$4 \quad$ Ver Jagannathan e Ma (2003).

5 Nesse caso, os métodos numéricos referem-se a algoritmos que aproximam numericamente as condições de primeira e segunda ordem que definem a solução do problema de otimização com restrições, uma vez que expressões analíticas não estão disponíveis para o problema. 


$$
\text { Turnover }=\frac{1}{L-T-1} \sum_{t=T}^{L-1} \sum_{j=1}^{N}\left(\left|w_{j, t+1}-w_{j, t}\right|\right) \text {. }
$$

A equação 18 calcula a média aritmética do retorno da carteira para os dados fora da amostra. De forma análoga, tem-se o desvio padrão na equação 19. Considerando que $L$ é o total de dados e $T$ o tamanho da janela de estimação, $L-T$ serão os dados fora da amostra, $w_{t}$ ' será o vetor de pesos dos ativos e $R_{t+1}$ será o retorno dos ativos um passo a frente. Pode-se observar que a medida de desempenho turnover pode ser uma proxy dos custos de rebalanceamento da carteira, pois leva em consideração as diferenças nas composições das carteiras entre os intervalos de tempo.

Para se mensurar o impacto dos custos de transação no desempenho das diferentes carteiras (HAN, 2006; DEMIGUEL; GARLAPPI; UPPAL, 2009; SANTOS et al., 2012), considera-se a média dos retornos líquidos de custos de transação, $\hat{\mu}_{\mathrm{CT}}$, dada por

$$
\hat{\mu}_{C T}=\frac{1}{L-T} \sum_{t=T}^{L-1}\left[\left(1+w_{t}^{\prime} R_{t+1}\right)\left(1-c \sum_{j=1}^{N}\left(\left|w_{j, t+1}-w_{j, t}\right|\right)-1\right)\right]
$$

em que c é a taxa a ser paga para cada transação. Ao invés de se supor um valor arbitrário para $c$, apresenta-se o valor de $c$ tal que a média do retorno líquido de custos de transação é igual a zero, conhecido como custo de transação breakeven. Vale observar que as estratégias que obtiverem custos breakeven mais altos são preferíveis, pois o custo de transação necessário para tornar essas estratégias isentas de lucro é mais alto.

Para testar a significância estatística das diferenças entre o desvio padrão e os índices de Sharpe da carteira de variância mínima em relação à carteira de mercado, adota-se uma estratégia similar a Caldeira, Moura e Santos (2013), que usam o bootstrap estacionário com $B=1000$ reamostragens e tamanho de bloco $b=5$. Os $p$-valores do teste foram obtidos usando a metodologia sugerida por Ledoit e Wolf (2008). Uma descrição da metodologia do bootstrap pode ser obtida nos trabalhos de Politis e Romano (1994) e Efron e Tibshirani (2004).

\section{Análise Empírica}

\subsection{Dados Utilizados e Descrição da Avaliação fora da Amostra}

A avaliação empírica da aplicabilidade dos modelos multivariados de volatilidade vistos na seção 2 é feita com base em uma avaliação fora da amostra utilizando-se em dois conjuntos de dados distintos. O primeiro conjunto contém retornos diários das 45 ações mais líquidas negociadas na bolsa de valores de São Paulo no período de $1^{\circ}$ de janeiro de 2008 a $1^{\circ}$ de janeiro de 2014 , totalizando 
1.489 observações. O segundo conjunto de dados contém retornos diários das 45 ações mais líquidas pertencentes ao índice norte-americano SEP100 entre $1^{\circ}$ de janeiro de 2008 e $1^{\circ}$ de janeiro de 2014, totalizando 1.510 observações. Em ambos os casos, os retornos foram calculados a partir da diferença entre o logaritmo dos preços. A taxa livre de risco para o primeiro grupo de dados foi a taxa do certificado de depósito interbancário (CDI) diário, enquanto para o segundo foi a taxa referente à T-Bill de um mês. As Tabelas 1 e 2 reportam estatísticas descritivas (retorno médio, desvio padrão dos retornos, assimetria e curtose) para os ativos que compõem as amostras brasileira e norte-americana, respectivamente. As tabelas indicam que os ativos reproduzem fatos estilizados na literatura de séries temporais financeiras, como, por exemplo, retorno médio próximo a zero e excesso de curtose em relação à distribuição gaussiana.

Tabela 1 - Estatísticas descritivas dos retornos diários da amostra de ações negociadas no mercado brasileiro

\begin{tabular}{lcccc}
\hline $\begin{array}{c}\text { Código do ativo } \\
\text { (ticker) }\end{array}$ & $\begin{array}{c}\text { Retorno } \\
\text { médio (\%) }\end{array}$ & $\begin{array}{c}\text { Desvio } \\
\text { padrão }\end{array}$ & Assimetria & Curtose \\
\hline BBAS3 & 0,016 & 2,576 & 0,142 & 9,015 \\
BBDC4 & 0,019 & 2,250 & 0,606 & 10,674 \\
BISA3 & $-0,136$ & 3,254 & $-0,119$ & 7,734 \\
BRAP4 & $-0,030$ & 2,714 & $-0,176$ & 8,287 \\
BRFS3 & 0,062 & 2,235 & 0,221 & 7,508 \\
BRKM5 & 0,035 & 2,746 & 0,428 & 6,513 \\
BRML3 & 0,029 & 2,995 & 0,567 & 12,990 \\
BTOW3 & $-0,103$ & 3,785 & 0,386 & 8,373 \\
CCRO3 & 0,080 & 2,219 & 0,254 & 10,659 \\
CESP6 & $-0,037$ & 2,891 & $-1,356$ & 23,752 \\
CMIG4 & 0,046 & 2,103 & $-1,094$ & 15,115 \\
CPFE3 & 0,035 & 1,793 & 0,034 & 7,905 \\
CPLE6 & 0,025 & 2,135 & $-0,080$ & 11,079 \\
CRUZ3 & 0,088 & 2,036 & 0,172 & 6,127 \\
CSNA3 & $-0,016$ & 3,026 & 0,151 & 8,011 \\
CYRE3 & $-0,028$ & 3,502 & 0,081 & 9,160 \\
DASA3 & 0,033 & 2,337 & 0,563 & 8,485 \\
ELET3 & $-0,051$ & 2,612 & 0,202 & 8,900 \\
EMBR3 & 0,005 & 2,503 & 0,071 & 6,962 \\
ENBR3 & 0,033 & 1,979 & $-0,135$ & 6,181 \\
\hline & & & & $60 n t i n u a \ldots$
\end{tabular}


conclusão.

\begin{tabular}{|c|c|c|c|c|}
\hline $\begin{array}{l}\text { Código do ativo } \\
\text { (ticker) }\end{array}$ & $\begin{array}{c}\text { Retorno } \\
\text { médio (\%) }\end{array}$ & $\begin{array}{l}\text { Desvio } \\
\text { padrão }\end{array}$ & Assimetria & Curtose \\
\hline GGBR4 & $-0,013$ & 2,836 & 0,060 & 6,396 \\
\hline GOAU4 & $-0,016$ & 2,860 & 0,104 & 6,324 \\
\hline GOLL4 & $-0,091$ & 3,692 & $-0,034$ & 7,607 \\
\hline HGTX3 & 0,159 & 2,805 & 0,302 & 10,556 \\
\hline ITSA4 & 0,027 & 2,455 & 0,675 & 11,454 \\
\hline ITUB4 & 0,020 & 2,478 & 0,654 & 10,748 \\
\hline JBSS3 & 0,029 & 3,454 & $-0,064$ & 7,983 \\
\hline LAME4 & 0,023 & 2,834 & 0,513 & 11,392 \\
\hline LREN3 & 0,049 & 2,945 & 0,119 & 7,615 \\
\hline MMXM3 & $-0,212$ & 5,058 & 0,545 & 16,081 \\
\hline MRFG3 & $-0,088$ & 3,198 & $-0,644$ & 10,467 \\
\hline MRVE3 & $-0,019$ & 3,855 & $-0,165$ & 8,658 \\
\hline NATU3 & 0,076 & 2,099 & 0,284 & 4,700 \\
\hline OIBR4 & $-0,062$ & 2,985 & 0,059 & 8,317 \\
\hline PCAR4 & 0,082 & 2,068 & 0,443 & 6,936 \\
\hline PDGR3 & $-0,075$ & 3,700 & $-0,110$ & 6,536 \\
\hline PETR4 & $-0,049$ & 2,497 & $-0,157$ & 7,023 \\
\hline RENT3 & 0,053 & 2,859 & $-0,081$ & 14,647 \\
\hline RSID3 & $-0,150$ & 3,915 & $-0,274$ & 5,937 \\
\hline SBSP3 & 0,057 & 2,500 & $-0,127$ & 7,682 \\
\hline SUZB5 & $-0,051$ & 2,653 & 0,150 & 5,370 \\
\hline TIMP3 & 0,029 & 2,934 & $-0,039$ & 16,505 \\
\hline USIM5 & $-0,033$ & 3,175 & 0,349 & 6,528 \\
\hline VALE5 & $-0,009$ & 2,445 & $-0,142$ & 8,139 \\
\hline VIVT4 & 0,036 & 1,727 & 0,039 & 4,932 \\
\hline Ibovespa & $-0,013$ & 1,933 & 0,056 & 9,953 \\
\hline
\end{tabular}

Fonte: Elaboração própria. 
Tabela 2 - Estatísticas descritivas dos retornos diários da amostra de ações negociadas no mercado norte-americano

\begin{tabular}{|c|c|c|c|c|}
\hline $\begin{array}{c}\text { Código do ativo } \\
\text { (ticker) }\end{array}$ & $\begin{array}{c}\text { Retorno } \\
\text { médio (\%) }\end{array}$ & $\begin{array}{l}\text { Desvio } \\
\text { padrão }\end{array}$ & Assimetria & Curtose \\
\hline $\mathrm{BAC}$ & $-0,057$ & 4,595 & $-0,232$ & 15,392 \\
\hline GE & $-0,003$ & 2,383 & 0,001 & 10,755 \\
\hline $\mathrm{F}$ & 0,059 & 3,499 & $-0,091$ & 16,089 \\
\hline S & $-0,013$ & 4,402 & $-0,473$ & 12,221 \\
\hline PFE & 0,037 & 1,609 & $-0,098$ & 8,641 \\
\hline WFC & 0,039 & 3,694 & 0,733 & 16,674 \\
\hline JPM & 0,030 & 3,368 & 0,291 & 12,677 \\
\hline $\mathrm{C}$ & $-0,110$ & 4,824 & $-0,379$ & 23,570 \\
\hline $\mathrm{T}$ & 0,012 & 1,563 & 0,647 & 14,876 \\
\hline $\mathrm{AA}$ & $-0,074$ & 3,311 & $-0,188$ & 9,689 \\
\hline EMC & 0,023 & 2,144 & 0,104 & 7,255 \\
\hline $\mathrm{XOM}$ & 0,015 & 1,757 & 0,144 & 18,677 \\
\hline $\mathrm{HPQ}$ & $-0,032$ & 2,369 & $-0,549$ & 13,784 \\
\hline $\mathrm{KO}$ & 0,032 & 1,340 & 0,617 & 14,438 \\
\hline $\mathrm{VZ}$ & 0,034 & 1,554 & 0,363 & 11,151 \\
\hline MRK & 0,008 & 1,860 & $-0,580$ & 13,469 \\
\hline $\mathrm{ABT}$ & 0,036 & 1,331 & $-0,186$ & 9,066 \\
\hline $\mathrm{HAL}$ & 0,023 & 2,994 & $-0,516$ & 9,344 \\
\hline WMT & 0,043 & 1,287 & 0,106 & 11,451 \\
\hline $\mathrm{MO}$ & 0,058 & 1,351 & $-0,172$ & 22,324 \\
\hline $\mathrm{HD}$ & 0,088 & 1,968 & 0,455 & 7,495 \\
\hline LOW & 0,061 & 2,241 & 0,293 & 7,147 \\
\hline COP & 0,019 & 2,118 & $-0,451$ & 11,670 \\
\hline $\mathrm{TXN}$ & 0,029 & 2,007 & $-0,498$ & 7,532 \\
\hline BMY & 0,065 & 1,587 & 0,079 & 7,207 \\
\hline JNJ & 0,035 & 1,120 & 0,586 & 16,933 \\
\hline PG & 0,020 & 1,252 & $-0,193$ & 10,246 \\
\hline DIS & 0,063 & 2,014 & 0,250 & 9,696 \\
\hline XRX & $-0,009$ & 2,591 & $-0,209$ & 10,794 \\
\hline DOW & 0,023 & 2,792 & $-0,395$ & 8,861 \\
\hline
\end{tabular}


conclusão.

\begin{tabular}{lcccc}
\hline $\begin{array}{c}\text { Código do ativo } \\
\text { (ticker) }\end{array}$ & $\begin{array}{c}\text { Retorno } \\
\text { médio (\%) }\end{array}$ & $\begin{array}{c}\text { Desvio } \\
\text { padrão }\end{array}$ & Assimetria & Curtose \\
\hline AXP & 0,045 & 2,999 & 0,061 & 10,790 \\
CVX & 0,033 & 1,916 & 0,178 & 17,810 \\
SLB & $-0,002$ & 2,677 & $-0,579$ & 10,155 \\
CVS & 0,044 & 1,865 & $-1,360$ & 24,105 \\
BK & $-0,012$ & 3,245 & $-0,066$ & 17,169 \\
CAT & 0,027 & 2,398 & 0,040 & 6,855 \\
WMB & 0,030 & 2,770 & $-0,427$ & 12,713 \\
TGT & 0,024 & 2,081 & 0,038 & 10,895 \\
WAG & 0,037 & 1,839 & 0,553 & 9,764 \\
MCD & 0,046 & 1,291 & $-0,055$ & 8,590 \\
PEP & 0,018 & 1,248 & $-0,430$ & 14,839 \\
DD & 0,042 & 2,080 & $-0,376$ & 7,993 \\
IBM & 0,046 & 1,519 & 0,019 & 8,380 \\
MDT & 0,019 & 1,687 & $-0,655$ & 11,027 \\
BA & 0,040 & 2,076 & 0,106 & 6,964 \\
\hline SP100 & $\mathbf{0 , 0 1 3}$ & $\mathbf{1 , 4 8 5}$ & $\mathbf{- 0 , 2 3 4}$ & $\mathbf{1 1 , 5 5 6}$ \\
\hline
\end{tabular}

Fonte: Elaboração própria.

Utiliza-se a metodologia de janelas móveis adotada em Santos (2010) para realizar uma avaliação de desempenho fora da amostra. Para ambas as amostras realizou-se o mesmo processo, o qual se baseia em uma janela de estimação de $T=252$ dias. A metodologia pode ser descrita da seguinte forma: primeiro, usando as primeiras $T$ observações de cada conjunto de dados, obteve-se a previsão um passo à frente da matriz de covariâncias condicionais segundo cada um dos modelos discutidos na seção 2; segundo, obtiveram-se as carteiras de mínima variância para cada uma das especificações da matriz de covariâncias condicionais. Terceiro, repetiu-se esse processo rolando a janela de estimação para frente de modo a eliminar a observação mais antiga e incluir a observação mais recente. Ao final desse processo, foram geradas $L-T$ observações fora da amostra para cada conjunto de dados, nos quais $L$ é o número total de observações. Todas as estatísticas de desempenho reportadas no artigo referem-se somente às observações fora da amostra. Finalmente, como forma de avaliar o impacto dos custos de transação e de rebalanceamento das carteiras, consideraram-se três frequências de rebalanceamento: diária, semanal e mensal. 


\subsection{Resultados para a Amostra Brasileira}

A Tabela 3 traz os resultados da avaliação do desempenho das carteiras de variância mínima para a amostra de ações do mercado acionário brasileiro. A tabela reporta, para cada especificação da matriz de covariâncias condicional e para cada frequência de rebalanceamento das carteiras, o retorno médio diário ao longo do período fora da amostra, o desvio padrão dos retornos, o índice de Sharpe, o turnover, o retorno acumulado bruto (RAB), o excesso de retorno acumulado (ERA) e o custo de transação breakeven. As equações 18 a 21 definem cada uma dessas estatísticas. Além disso, a Tabela 3 indica com asteriscos os casos em que o desvio padrão e o índice de Sharpe são estatisticamente diferentes daqueles obtidos pela carteira de mercado (índice Ibovespa).

Tabela 3 - Amostra brasileira: desempenho fora da amostra das carteiras de mínima variância obtidas com diferentes especificações Garch multivariadas para as matrizes de covariâncias condicionais dos retornos

\begin{tabular}{|c|c|c|c|c|c|c|c|}
\hline & $\begin{array}{c}\text { Retorno } \\
\text { médio } \\
\text { (\%) }\end{array}$ & $\begin{array}{c}\text { Desvio } \\
\text { padrão } \\
(\%)\end{array}$ & $\begin{array}{c}\text { Índice } \\
\text { de } \\
\text { Sharpe }\end{array}$ & $\begin{array}{l}\text { Turn } \\
\text { over }\end{array}$ & $\begin{array}{c}\text { RAB } \\
\text { (\%) }\end{array}$ & $\begin{array}{c}\text { ERA } \\
\text { (\%) }\end{array}$ & $\begin{array}{c}\text { Custo } \\
\text { break- } \\
\text { even }\end{array}$ \\
\hline \multicolumn{8}{|c|}{ Rebalanceamento diário } \\
\hline $\begin{array}{l}\text { CCC- } \\
\text { GARCH }\end{array}$ & 0,064 & $0,999^{*}$ & $0,064^{*}$ & 0,299 & 107,621 & 32,681 & 20,178 \\
\hline $\begin{array}{l}\text { DCC- } \\
\text { GARCH }\end{array}$ & 0,037 & $1,188^{*}$ & 0,031 & 1,043 & 45,136 & $-7,263$ & 3,438 \\
\hline $\begin{array}{l}\text { DECO- } \\
\text { GARCH }\end{array}$ & 0,046 & $1,004^{*}$ & 0,046 & 0,307 & 65,904 & 6,012 & 14,344 \\
\hline $\begin{array}{l}\text { ASY- } \\
\text { DCC- } \\
\text { GARCH }\end{array}$ & 0,072 & $0,963^{*}$ & $0,075^{*}$ & 0,233 & 129,604 & 46,734 & 28,728 \\
\hline \multicolumn{8}{|c|}{ Rebalanceamento semanal } \\
\hline $\begin{array}{l}\text { CCC- } \\
\text { GARCH }\end{array}$ & 0,061 & $1,023^{*}$ & $0,060^{*}$ & 0,124 & 99,474 & 27,470 & 46,272 \\
\hline $\begin{array}{l}\text { DCC- } \\
\text { GARCH }\end{array}$ & 0,055 & $1,186^{*}$ & 0,046 & 0,305 & 79,833 & 14,916 & 16,977 \\
\hline $\begin{array}{l}\text { DECO- } \\
\text { GARCH }\end{array}$ & 0,041 & $1,019^{*}$ & 0,041 & 0,129 & 56,578 & 0,050 & 30,853 \\
\hline $\begin{array}{l}\text { ASY- } \\
\text { DCC- } \\
\text { GARCH }\end{array}$ & 0,071 & $1,022^{*}$ & $0,070^{*}$ & 0,099 & 126,348 & 44,650 & 67,309 \\
\hline
\end{tabular}




\begin{tabular}{ccccccc}
$\begin{array}{c}\text { Retorno } \\
\text { médio }\end{array}$ & $\begin{array}{c}\text { Desvio } \\
\text { padrão }\end{array}$ & $\begin{array}{c}\text { Índice } \\
\text { de } \\
(\%)\end{array}$ & $\begin{array}{c}\text { Turn } \\
\text { over }\end{array}$ & $\begin{array}{c}\text { RAB } \\
(\%)\end{array}$ & $\begin{array}{c}\text { ERA } \\
(\%)\end{array}$ & $\begin{array}{c}\text { Custo } \\
\text { break- } \\
\text { even }\end{array}$ \\
\hline
\end{tabular}

\begin{tabular}{|c|c|c|c|c|c|c|c|}
\hline \multicolumn{8}{|c|}{ Rebalanceamento mensal } \\
\hline $\begin{array}{l}\text { CCC- } \\
\text { GARCH }\end{array}$ & 0,059 & $1,059 *$ & $0,056 *$ & 0,051 & 94,127 & 24,054 & 109,505 \\
\hline $\begin{array}{l}\text { DCC- } \\
\text { GARCH }\end{array}$ & 0,033 & $1,214^{*}$ & 0,027 & 0,089 & 37,524 & $-12,130$ & 36,172 \\
\hline $\begin{array}{l}\text { DECO- } \\
\text { GARCH }\end{array}$ & 0,027 & $1,059 *$ & 0,026 & 0,052 & 30,792 & $-16,432$ & 51,308 \\
\hline $\begin{array}{l}\text { ASY- } \\
\text { DCC- } \\
\text { GARCH }\end{array}$ & 0,071 & $1,060 *$ & $0,067^{*}$ & 0,043 & 123,691 & 42,953 & 152,616 \\
\hline
\end{tabular}

\begin{tabular}{llllll}
\hline Ibovespa & $-0,020$ & 1,503 & $-0,014$ & 5,857 & $-32,372$ \\
\hline
\end{tabular}

Fonte: Elaboração própria.

Nota: As estatísticas de retorno e desvio padrão são baseadas em retornos diários. O asterisco indica que os valores são estatisticamente diferentes ao nível de significância de 5\% em relação aos obtidos com a carteira de mercado (índice Ibovespa).

Os resultados da Tabela 3 indicam que todas as especificações Garch multivariadas geraram carteiras de variância mínima com risco (desvio padrão) estatisticamente inferior ao obtido pelo índice Ibovespa em todas as frequências de rebalanceamento. Para um rebalanceamento diário das carteiras, o melhor resultado em termos de desvio padrão foi obtido pelo modelo ASYDCC-GARCH $(0,963)$, seguido pelo modelo CCC-GARCH $(0,999)$. Além disso, verificou-se que o retorno ajustado ao risco medido pelo índice de Sharpe foi superior ao obtido pelo índice Ibovespa em vários casos. O maior índice de Sharpe foi obtido pelo modelo ASYDCC-GARCH $(0,075)$, seguido pelo modelo CCC-GARCH $(0,064)$ sob rebalanceamento diário. Em termos de retorno acumulado, novamente os modelos ASYDCC-GARCG e CCC-GARCH obtiveram um desempenho substancialmente superior ao índice de mercado, alcançando um RAB de 129\% e 107\%, respectivamente, e um ERA de $46 \%$ e $32 \%$, respectivamente. No mesmo período, esses números para o índice Ibovespa são, respectivamente, $6 \%$ e - $32 \%$.

Verificou-se também que a redução da frequência de rebalanceamento traz uma redução substancial no custo de transação medido pelo turnover. Conforme esperado, verificou-se que uma maior frequência de rebalanceamento está associada a um maior turnover das carteiras de mínima variância. A especificação DCC-GARCH obteve maior turnover ao longo de todas as frequências de rebalanceamen- 
to, enquanto a especificação ASYDCC-GARCH obteve menor turnover em todos os casos. Entretanto, a redução na frequência de rebalanceamento acarretou em alguns casos uma deterioração do desempenho das carteiras em termos de retorno ajustado ao risco. Por exemplo, o índice de Sharpe das carteiras de variância mínima obtidas com o modelo ASYDCC-GARCH foi de 0,075 , para uma frequência de rebalanceamento diária, e de 0,067 para uma frequência mensal. Entretanto, esse último índice de Sharpe ainda é estatisticamente superior ao obtido pelo índice Ibovespa, o que indica que mesmo em um contexto de baixa frequência de rebalanceamento é possivel conseguir retornos ajustados ao risco superiores ao obtido pela carteira de mercado.

A análise do custo breakeven sugere que é necessário um custo de transação elevado para tornar o lucro líquido de custos de transação igual a zero. Por exemplo, o custo breakeven das carteiras de variância mínima obtidas com o modelo ASYDCC-GARCH sob rebalanceamento mensal é de 152 pontos-base. Resultados semelhantes são obtidos para os demais modelos, sugerindo que a implementação de carteiras de variância mínima com base em modelos de correlações condicionais é robusta do ponto de vista dos custos de transação.

O Gráfico 1 traz o RBA de cada um dos modelos multivariados de volatilidade ao longo do período fora da amostra, além do RBA do índice Ibovespa e da taxa livre de risco. O gráfico indica que todos os modelos multivariados de volatilidade geraram carteiras de variância mínima com retorno acumulado superior ao Ibovespa ao longo de todo o período fora da amostra. Além disso, com exceção do modelo DCC-GARCH, todas as especificações geraram carteiras com RBA superior ao RBA do ativo livre de risco. 


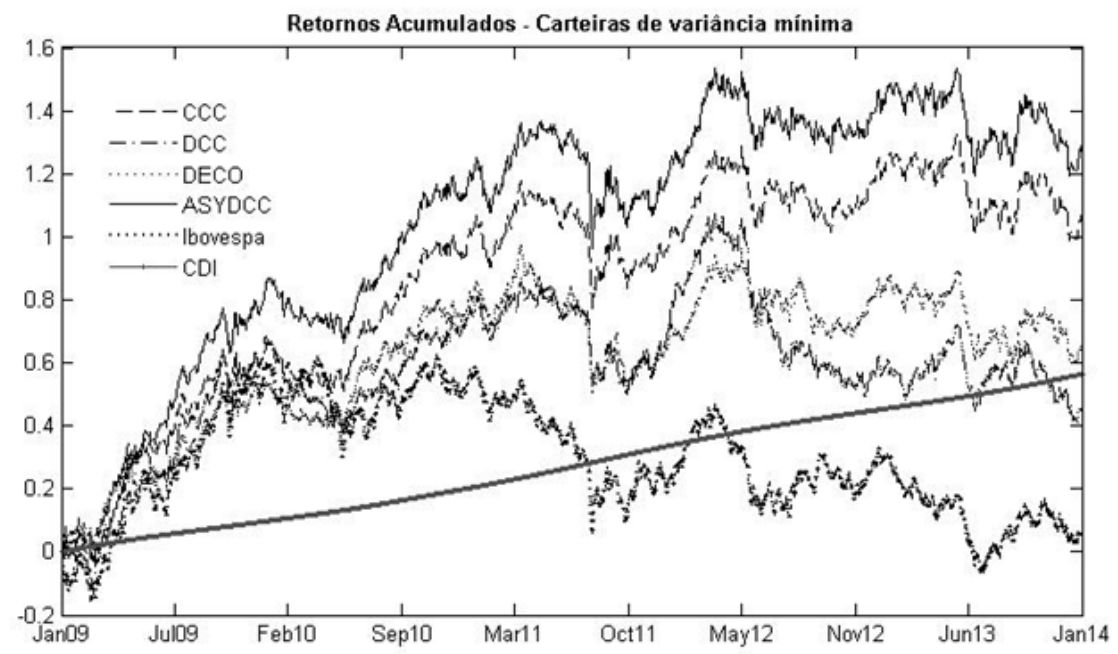

Fonte: Elaboração própria.

Nota: CDI indica taxa livre de risco.

\subsection{Resultados para a Amostra Norte-Americana}

A Tabela 4 traz os resultados da avaliação do desempenho fora da amostra das carteiras de variância mínima para a amostra de ações do mercado norte-americano. A tabela reporta para cada especificação da matriz de covariâncias condicional e para cada frequência de rebalanceamento das carteiras o retorno médio diário das carteiras ao longo do período fora da amostra, o desvio padrão, o índice de Sharpe, o turnover, o RAB e o ERA e o custo de transação breakeven. Além disso, a tabela indica com asteriscos os casos em que o desvio padrão e o índice de Sharpe são estatisticamente diferentes dos obtidos pela carteira de mercado. 
Tabela 4 - Amostra norte-americana: desempenho fora da amostra das carteiras de mínima variância obtidas com diferentes especificações Garch multivariadas para as matrizes de covariâncias condicionais dos retornos

\begin{tabular}{|c|c|c|c|c|c|c|c|}
\hline & $\begin{array}{c}\text { Retorno } \\
\text { médio } \\
(\%)\end{array}$ & $\begin{array}{c}\text { Desvio } \\
\text { padrão } \\
(\%)\end{array}$ & $\begin{array}{c}\text { Indice } \\
\text { de } \\
\text { Sharpe }\end{array}$ & $\begin{array}{l}\text { Turn } \\
\text { over }\end{array}$ & $\begin{array}{c}\text { RAB } \\
(\%)\end{array}$ & $\begin{array}{c}\text { ERA } \\
(\%)\end{array}$ & $\begin{array}{c}\text { Custo } \\
\text { break- } \\
\text { even }\end{array}$ \\
\hline \multicolumn{8}{|c|}{ Rebalanceamento diário } \\
\hline $\begin{array}{l}\text { CCC- } \\
\text { GARCH }\end{array}$ & 0,057 & $0,766 *$ & 0,074 & 0,350 & 97,416 & 96,694 & 15,412 \\
\hline $\begin{array}{l}\text { DCC- } \\
\text { GARCH }\end{array}$ & 0,048 & $0,863^{*}$ & 0,056 & 0,515 & 74,455 & 73,816 & 8,891 \\
\hline $\begin{array}{l}\text { DECO- } \\
\text { GARCH }\end{array}$ & 0,052 & $0,779 *$ & 0,067 & 0,340 & 85,218 & 84,540 & 14,543 \\
\hline $\begin{array}{l}\text { ASY- } \\
\text { DCC- } \\
\text { GARCH }\end{array}$ & 0,063 & $0,765^{*}$ & $0,082 *$ & 0,302 & 112,014 & 111,238 & 19,540 \\
\hline \multicolumn{8}{|c|}{ Rebalanceamento semanal } \\
\hline $\begin{array}{l}\text { CCC- } \\
\text { GARCH }\end{array}$ & 0,060 & $0,767^{*}$ & $0,078 *$ & 0,139 & 104,062 & 103,316 & 40,506 \\
\hline $\begin{array}{l}\text { DCC- } \\
\text { GARCH }\end{array}$ & 0,053 & $0,855^{*}$ & 0,062 & 0,187 & 86,212 & 85,531 & 27,000 \\
\hline $\begin{array}{l}\text { DECO- } \\
\text { GARCH }\end{array}$ & 0,057 & $0,786 *$ & 0,073 & 0,140 & 97,635 & 96,912 & 38,804 \\
\hline $\begin{array}{l}\text { ASY- } \\
\text { DCC- } \\
\text { GARCH }\end{array}$ & 0,059 & $0,782 *$ & $0,076 *$ & 0,117 & 102,907 & 102,164 & 47,742 \\
\hline
\end{tabular}

continua... 
conclusão.

\begin{tabular}{|c|c|c|c|c|c|c|c|}
\hline & $\begin{array}{c}\text { Retorno } \\
\text { médio } \\
(\%)\end{array}$ & $\begin{array}{c}\text { Desvio } \\
\text { padrão } \\
(\%)\end{array}$ & $\begin{array}{c}\text { Indice } \\
\text { de } \\
\text { Sharpe }\end{array}$ & $\begin{array}{l}\text { Turn } \\
\text { over }\end{array}$ & $\begin{array}{c}\text { RAB } \\
(\%)\end{array}$ & $\begin{array}{c}\text { ERA } \\
(\%)\end{array}$ & $\begin{array}{c}\text { Custo } \\
\text { break- } \\
\text { even }\end{array}$ \\
\hline \multicolumn{8}{|c|}{ Rebalanceamento mensal } \\
\hline $\begin{array}{l}\text { CCC- } \\
\text { GARCH }\end{array}$ & 0,058 & $0,785^{*}$ & 0,074 & 0,050 & 100,242 & 99,509 & 110,288 \\
\hline $\begin{array}{l}\text { DCC- } \\
\text { GARCH }\end{array}$ & 0,073 & $0,921 *$ & $0,080 *$ & 0,066 & 138,365 & 137,493 & 103,374 \\
\hline $\begin{array}{l}\text { DECO- } \\
\text { GARCH }\end{array}$ & 0,055 & $0,804^{*}$ & 0,069 & 0,050 & 92,003 & 91,301 & 103,711 \\
\hline $\begin{array}{l}\text { ASY- } \\
\text { DCC- } \\
\text { GARCH }\end{array}$ & 0,068 & $0,791 *$ & $0,086 *$ & 0,045 & 126,793 & 125,963 & 142,907 \\
\hline
\end{tabular}

\begin{tabular}{llllll}
\hline SP100 & 0,051 & 1,167 & 0,044 & 75,212 & 74,570 \\
\hline
\end{tabular}

Fonte: Elaboração própria.

Nota: As estatísticas de retorno e desvio padrão são baseadas em retornos diários. O asterisco indica que os valores são estatisticamente diferentes ao nível de significância de $5 \%$ em relação aos obtidos com a carteira de mercado (índice SEP100).

Os resultados para o mercado norte-americano são semelhantes aos obtidos para o mercado brasileiro. Os números da Tabela 4 indicam que todas as especificações Garch multivariadas consideradas no artigo geraram carteiras de mínima variância com risco (desvio padrão) substancialmente e estatisticamente inferior ao obtido pela carteira de mercado ao longo de todas as frequências de rebalanceamento. O melhor resultado foi obtido pela especificação ASYDCC-GARCH com frequência de rebalanceamento diária (desvio padrão de 0,765 versus 1,167 da carteira de mercado dada pelo índice SEP100). Em termos de retornos ajustados ao risco, constatou-se que a especificação ASYDCC-GARCH gerou carteiras de mínima variância com índice de Sharpe estatisticamente diferente (superior) àquele obtido pela carteira de mercado ao longo de todas as frequências de rebalanceamento. Em termos de retorno acumulado, verificou-se que na vasta maioria dos casos as especificações geraram um RBA positivo e superior àquele da carteira de mercado. O melhor resultado em termos de RBA foi alcançado pela especificação ASYDCC-GARCH com rebalanceamento mensal (126\%), o que é substancialmente maior que o RBA alcançado pelo índice SEP100 (75\%). Além disso, o ERA obtido por todas as especificações foi positivo e superior àquele obtido pela carteira de mercado ao longo de todas as frequências de rebalanceamento. 
Ao contrário dos resultados obtidos com a amostra brasileira, verificou-se na Tabela 4 que a diminuição da frequência de rebalanceamento das carteiras de mínima variância não acarretou uma deterioração do desempenho das carteiras em termos de desvio padrão e índice de Sharpe. Ao contrário, observou-se que o melhor desempenho em termos de desvio padrão foi obtido no caso de rebalanceamento mensal. Conforme esperado, observou-se também que a redução na frequência de rebalanceamento acarretou uma redução expressiva nos custos de transação medidos pelo turnover.

Por sua vez, a análise do custo breakeven indica que é necessário um custo de transação elevado para tornar o lucro líquido de custos de transação igual a zero. Por exemplo, o custo breakeven das carteiras de variância mínima obtidas com o modelo ASYDCC-GARCH sob rebalanceamento mensal é de 142 pontos-base. Resultados semelhantes são obtidos para os demais modelos, sugerindo que a implementação de carteiras de variância mínima para o mercado norte-americano com base em modelos de correlações condicionais também é robusta do ponto de vista dos custos de transação.

O Gráfico 2 traz o RBA de cada um dos modelos multivariados de volatilidade ao longo do período fora da amostra, além do RBA do índice SEP100 e da taxa livre de risco (T-Bill). O gráfico indica que todos os modelos multivariados de volatilidade geraram carteiras de variância mínima com retorno acumulado superior ao índice S\&P100 e superior à taxa livre de risco ao longo de todo o período fora da amostra.

Gráfico 2 - Amostra norte-americana: RBA (rebalanceamento diário)

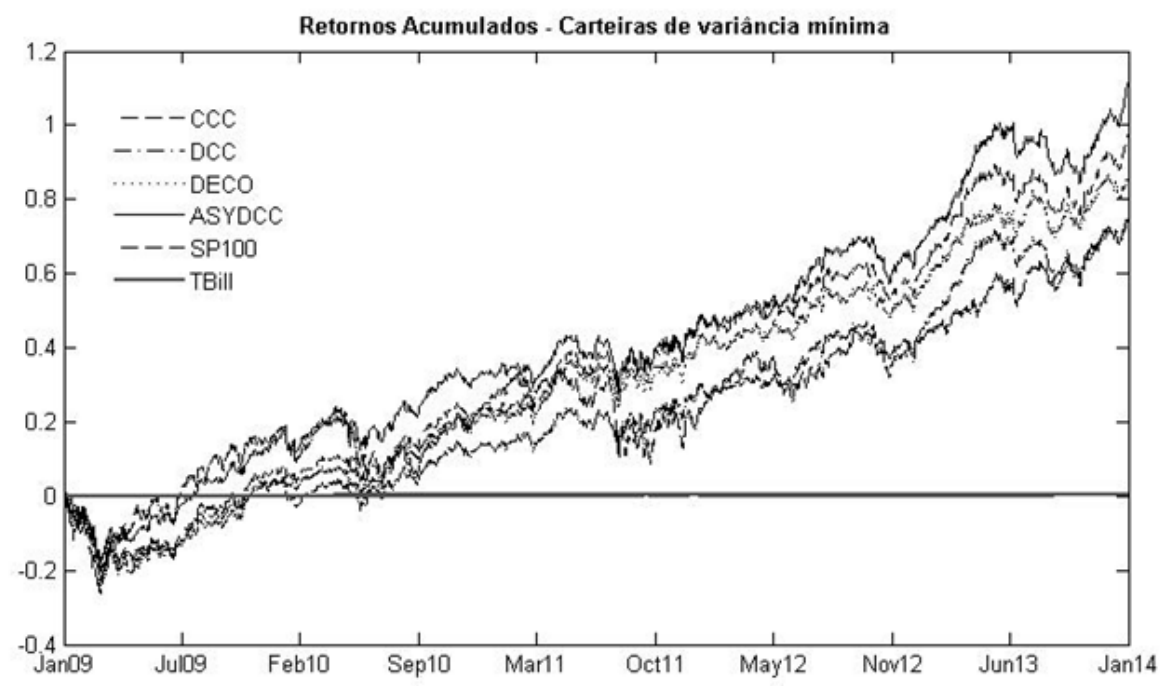

Fonte: Elaboração própria. 


\subsection{Discussão}

Os resultados discutidos nas seções 4.2 e 4.3 permitem uma discussão que aborda ao menos dois aspectos principais: primeiro, é possível concluir que modelos Garch multivariados da classe de correlações condicionais são capazes de ser aplicados em problemas de grande dimensão. A análise empírica feita neste artigo, baseada em duas amostras de 45 ativos, pode ser considerada desafiadora, pois o número de elementos estimados em cada matriz de covariâncias condicional é elevado. Sem embargo, os resultados obtidos para ambas as amostram revelam que as matrizes de covariâncias obtidas com essas especificações possuem validade empírica, pois geraram carteiras de variância mínima com desempenho fora da amostra superior ao observado pelos respectivos índices de referência de mercado. Esse resultado corrobora uma série de evidências empíricas anteriores como, por exemplo, as obtidas por Jagannathan e Ma (2003), entre outros.

O segundo ponto que merece ser discutido diz respeito à comparação entre os resultados obtidos para cada um dos países. Os resultados são bastante homogêneos para ambos os países e apontam para a superioridade da especificação ASYDCC-GARCH ao longo de todas as frequências de rebalanceamento estudadas. Esses resultados apontam para uma preferência em relação aos modelos de correlações condicionais dinâmicas em detrimento do modelo de correlação condicional constante além da preferência por modelos de correlações assimétricas em detrimento do modelo de correlações simétricas. Nesse sentido, os resultados encontrados no artigo corroboram a evidência anterior encontrada em Engle e Colacito (2006).

\section{Considerações Finais}

A modelagem da matriz de covariâncias condicional dos retornos de ativos financeiros através de modelos Garch multivariados é um tópico central na literatura especializada em economia e econometria financeira. A maioria das tentativas iniciais de se construir modelos para as covariâncias condicionais como, por exemplo, o modelo Vech de Bollerslev, Engle e Wooldridge (1988), e o modelo Bekk, de Engle e Kroner (1995), entre outros, sofrem da chamada maldição da dimensionalidade. Nessas especificações, o número de parâmetros cresce rapidamente à medida que a dimensão do problema aumenta, criando dificuldades no processo de estimação e introduzindo erros de estimação nas matrizes de covariâncias estimadas. Dessa forma, os modelos de correlações condicionais introduzidos por Bollerslev (1990) emergem como alternativas mais parcimoniosas e que requerem uma quantidade menor de parâmetros a serem estimados. 
Este artigo buscou implementar e avaliar o desempenho de modelos Garch multivariados da classe de correlações condicionais para a obtenção de carteiras de variância mínima envolvendo um grande número de ativos. Para isso, consideram-se as seguintes especificações: o modelo de correlações condicionais constantes (CCC-GARCH), de Bollerslev (1990), o modelo de correlações condicionais dinâmicas (DCC-GARCH), de Engle (2002), o modelo de equicorrelações condicionais dinâmicas (DECO-GARCH), de Engle e Kelly (2012), e o modelo correlações condicionais assimétricas (ASYDCC-GARCH), de Cappiello, Engle e Sheppard (2006). A escolha do problema da carteira de mínima variância se deve à importância que essa estratégia tem recebido na literatura recente. Essa estratégia depende exclusivamente das estimativas de covariâncias e, portanto, é menos vulnerável ao erro de estimação em comparação ao paradigma da média variância introduzido originalmente por Markowitz (1952). Além disso, evidências empíricas anteriores mostram que a carteira de mínima variância tem melhor desempenho fora da amostra do que qualquer outra carteira de média variância, mesmo quando se utilizam medidas de desempenho que dependem tanto da média quanto da variância.

A avaliação empírica realizada no artigo se baseia em dois conjuntos de dados distintos: o primeiro conjunto de dados contém as 45 ações de maior liquidez negociadas no mercado acionário brasileiro, enquanto o segundo é composto por 45 ativos de maior liquidez pertencentes ao índice SESP100 do mercado acionário norte-americano. Os resultados encontrados neste artigo depõem favoravelmente ao uso dos modelos multivariados de volatilidade em ambos os casos. Foi verificado que o risco das carteiras de variância mínima obtida com os modelos multivariados de volatilidade foi substancialmente e estatisticamente inferior ao obtido pelos índices de referência de ambos os mercados. Além disso, o retorno ajustado ao risco das carteiras de mínima variância obtida com a vasta maioria das especificações adotadas no artigo foi estatisticamente superior àquela obtida pelo índice de mercado usado como referência. Mais especificamente, os resultados apontam para uma preferência dos modelos de correlações condicionais dinâmicas em detrimento do modelo de correlação condicional constante, além da preferência por modelos de correlações assimétricas em detrimento do modelo de correlações simétricas.

\section{Referências}

BOLLERSLEV, T. Modeling the coherence in short-run nominal exchange rates: a multivariate generalized ARCH model. Review of Economics and Statistics, v. 72, n. 3, p. 498-505, 1990.

BOLLERSLEV, T.; ENGLE, R.; WOOLDRIDGE, J. A capital asset pricing model with timevarying covariances. Journal of Political Economy, v. 96, n. 1, p. 116-131, 1988. 
BRANDT, M. Portfolio choice problems. In: AIT-SAHALIA, Y.; HANSEN, L.P. (Org.). Handbook of Financial Econometrics, volume 1: tools and techniques, Amsterdam: North Holland, 2010. p. 269-336.

CALDEIRA, J. et al. Seleção de carteiras com modelos fatoriais heterocedásticos: aplicação para fundos de fundos multimercados. Revista de Administração Mackenzie, v. 15, n. 2, p. 127-161, 2014

CALDEIRA, J.; MOURA, G.; SANTOS, A. Seleção de carteiras usando o modelo FamaFrench-Carhart. Revista Brasileira de Economia, v. 67, n. 1, p. 45-65, 2013.

CAPPIELLO, L.; ENGLE, R.; SHEPPARD, K. Asymmetric dynamics in the correlations of global equity and bond returns. Journal of Financial Econometrics, v. 4, n. 4, p. 537-572, 2006.

DEMIGUEL, V.; GARLAPPI, L.; UPPAL, R. Optimal versus naive diversification: how inefficient is the 1/N portfolio strategy? Review of Financial Studies, v. 22, n. 5, p. 1915-1953, 2009.

EFRON, B.; TIBSHIRANI, R. An introduction to the bootstrap. Boca Raton: CRC Press, 1994.

ENGLE, R. Dynamic conditional correlation: a simple class of multivariate generalized autoregressive conditional heteroskedasticity models. Journal of Business and Economic Statistics, v. 20, n. 3, p. 339-350, 2002.

ENGLE, R.; COLACITO, R. Testing and valuing dynamic correlations for asset allocation. Journal of Business and Economic Statistics, v. 24, n. 2, p. 238-253, 2006.

ENGLE, R.; KELLY, B. Dynamic equicorrelation. Journal of Business 8 Economic Statistics, v. 30, n. 2, p. 212-228, 2012.

ENGLE, R.; KRONER, K. Multivariate simultaneous GARCH. Econometric Theory, v. 11, n. 1, p. 122-150, 1995.

ENGLE, R.; NG, V.; ROTHSCHILD, M. Asset pricing with a factor-ARCH covariance structure: Empirical estimates for treasury bills. Journal of Econometrics, v. 45, n. 1, p. 213-237, 1990.

ENGLE, R.; SHEPHARD, N.; SHEPPARD, K. Fitting vast dimensional time-varying covariance models. Oxford: Department of Economics, University of Oxford, 2008. (Discussion Paper Series, n. 403).

FLEMING, J.; KIRBY, C.; OSTDIEK, B. The economic value of volatility timing. Journal of Finance, v. 56, n. 1, p. 329-352, 2001.

HAN, Y. Asset allocation with a high dimensional latent factor stochastic volatility model. Review of Financial Studies, v. 19, n. 1, p. 237-271, 2006.

JAGANNATHAN, R.; MA, T. Risk reduction in large portfolios: why imposing the wrong constraints helps. The Journal of Finance, v. 58, n.4, p. 1651-1684, 2003.

LANNE, M.; SAIKKONEN, P. A multivariate generalized orthogonal factor GARCH model. Journal of Business and Economic Statistics, v. 25, n. 1, p. 61-75, 2007. 
LEDOIT, O.; WOLF, M. A well-conditioned estimator for large-dimensional covariance matrices, Journal of Multivariate Analysis, v. 88, n. 2, p. 365-411, 2004.

. Robust performance hypothesis testing with the Sharpe ratio. Journal of Empirical Finance, v. 15, n. 5, p. 850-859, 2008.

MARKOWITZ, H. Portfolio selection. Journal of Finance, v. 7, n. 1, p. 77-91, 1952.

POLITIS, D.; ROMANO, J. The stationary bootstrap. Journal of the American Statistical Association, v. 89, n. 428, p. 1303-1313, 1994.

RUBESAM, A.; BELTRAME, A. Carteiras de variância mínima no Brasil. Revista Brasileira de Finanças, v. 11, n. 1, p. 81-118, 2013.

SANTOS, A. The out of sample performance of robust portfolio optimization. Revista Brasileira de Finanças, v. 8, n. 2, p. 141-166, 2010.

SANTOS, A. et al. Optimal portfolios with minimum capital requirements. Journal of Banking and Finance, v, 36, n. 7, p. 1928-1942, 2012.

SANTOS, A.; MOURA, G. Dynamic factor multivariate GARCH model. Computational Statistics and Data Analysis, v. 76, p. 606-617, 2014.

SANTOS, A.; TESSARI, C. Técnicas quantitativas de otimização de carteiras aplicadas ao mercado de ações brasileiro. Revista Brasileira de Finanças, v. 10, n. 3, p. 369-393, 2012.

SILVENNOINEN, A.; TERASVIRTA, T. Multivariate GARCH models. In: ANDERSEN, T.G. et al. (Org.). Handbook of Financial Time Series. Berlin: Springer Verlag, 2009. p. 221-229.

THOMÉ, C.; LEAL, R.; ALMEIDA, V. Um índice de mínima variância de ações brasileiras. Economia Aplicada, v. 15, n. 4, p. 535-557, 2011.

TSE, Y. K.; TSUI, K. C. A multivariate generalized autoregressive conditional heteroscedasticity model with time-varying correlations. Journal of Business and Economic Statistics, v. 20, n. 3, p. 351-362, 2002.

VAN DER WEIDE, R. GO-GARCH: A Multivariate Generalized Orthogonal GARCH Model. Journal of Applied Econometrics, v. 17, n. 5, p. 549-564, 2002.

Recebido em: 07/10/2013. Aceito em: 04/09/2014. 